\title{
Evaluating Website Resources
}

Helen Hough, MLS, BS, BA

This manuscript version

is a rendition of

the author's post review copy.

The official version of record

is available at the

Perioperative Nursing Clinics site.
Nursing Informatics

\section{KEYWORDS}

- Webpages $\bullet$ Information resource evaluation $\bullet$ Information management $\bullet$ Internet

- Decision making

\section{KEY POINTS}

- The evaluation of websites can be made easier by using relevancy and reliability criteria.

- The contents of webpages are relevant when they meet the needs of the viewers in terms of content purpose, coverage, language, and timeliness.

- Reliability can be assessed when there is information about the author and hosting site along with contact data, good spelling and grammar, and the date of creation.

- Most importantly, viewers have to trust their knowledge and determine if the information is accurate, objective, and supported by appropriate references and links.

- Use of a tool can make systematic evaluation of websites consistent, particularly when these resources are needed as information support in a perioperative nursing project.

At many workplaces there is one person known for sharing interesting bits of information by forwarding e-mails or bringing in newspaper and Internet articles. While cartoons and jokes may be amusing, sometimes the information is composed of doubtful facts, including terrible crime threats, political scandals, and unusual medical treatments. The same stories that might have been kindly dismissed when discussed in the break room are somehow thought to be more substantial and reliable because they are in print. Casually judging the value of these stories is easy when the outcome is unimportant. The evaluation of information is more difficult when the outcome is more significant, such as when people's health or lives are involved. The provision of good health care should be supported by current, reliable, and authoritative information. Evaluating sources is an important health care skill.

Words on paper or on a webpage are just data if no one reads the material. Data has little value until it is understood and used by an individual. Data becomes

The author has nothing to disclose.

Science and Engineering Library, University of Texas at Arlington, PO Box 19497, B03

Nedderman Hall, 416 Yates Street, Arlington, TX 76019, USA

E-mail address: hough@uta.edu

Perioperative Nursing Clinics 7 (2012) 189-193

doi:10.1016/j.cpen.2012.02.007

periopnursing.theclinics.com

(C) 2012 Elsevier Inc. All rights reserved. 
information only when a person has read and understood its potential significance. Knowledge is the ability to recall and use information as input into decisions. ${ }^{1}$ Nurses may become aware of volumes of information as more people use the Internet for communication. A mere 150 years ago, the only reliable way to communicate across large distances was by the written word. While technology may have changed the speed, form, and volume of information being transferred, people are not significantly different. We still need reliable information to solve physical and social problems. The assessment of information provided by others has always been based on the context of social structures and values.

\section{SOCIAL CONSTRAINTS OF INFORMATION}

The concept of the suitability of information is based in its utility. By definition, data does not become information unless it is understood by the user. ${ }^{2}$ Language and tone affect emotional responses to information, and emotions affect comprehension. Relevancy is also a component of suitability. Informed decisions can only be made when the information is relevant to the issue at hand. User expectations, the match between available data and what is needed, timeliness, and the focus of the information affect relevancy. ${ }^{3}$ An information user is better able to judge the suitability of sources if the original question or need is understood.

The concept of authority in information is part of a social structure. Patients and colleagues look to nurses and other health care providers as knowledgeable information providers, valuing the expertise of these professionals. Expertise is assessed through awareness of length of practice, professional credentials, place of employment, relevancy of the expert's prior work, and recognition by other professionals.

The methods of evaluating printed materials can be compared to judging the value of information transmitted orally. For example, more questions may arise regarding the reliability of health care research information relayed by a teenage supermarket cashier than there would be if the same materials are discussed in a professional seminar by a scientist on the research team. Personnel facts transmitted by the chief executive officer of the hospital may be assessed as more valuable than those told by a neighbor employed in a different profession. The first example is one of authority and the second, of relevancy. Resources in print or from the Internet can be assessed in a similar manner. Most reliable documents can be obtained from recognized organizations' websites or can be checked for authors' names, credentials, and affiliations. Responsible journals and books are published by known and respected publishers. A wise person considers how any information is obtained and judges its value based on similar criteria regardless of format.

\section{CRITERIA FOR ASSESSING INFORMATION SOURCES}

Understand the need for the information. It is important to appreciate the variables that need to be addressed by this information and the impact of the information itself. A document, video, or other resource may not be relevant if the amount or language of the information inappropriate. The coverage may be too broad or narrow. The language may be too scholarly or elementary. When examining a document for suitability, consider when it was created and its intended audience. Authors write with specific audiences in mind, and material is less relevant if the document content and the audience do not correspond. An example of audience mismatch may be standard educational materials written at a high school level being provided to a patient requiring low-literacy information. Attitudes and health care knowledge also change, and it is important to be aware of when materials were originally produced. 
After the need and use of the information is understood, the materials themselves can be assessed. Authority, objectivity, documentation, and verifiability are common assessment variables. Some of the most important aspects of reliability are relatively simple to assess. If there are multiple spelling and grammar errors, the content may have additional problems in the accuracy of facts or other content. If there is no indication of how to contact the author or website for clarification, these errors cannot be challenged or corrected. Responsible authors take care in how their work is presented. They also provide a means of contact for feedback and corrections. An example of suspect reliability is Wikipedia; the contributors remain anonymous and do not have to provide contact information to the organization. Anonymous sources like Wikipedia entries can be used as a beginning discovery tool for basic terms and concepts but should not be considered authoritative.

Authority usually is judged by determining if the author or organization that produced the material is reliable. Scholarly resources are written by those with appropriate academic credentials. Sometimes an author is not specified. In these cases, an organization or agency accepts responsibility for the content. Sponsorship from a reputable organization or being posted on the organization's website carries weight similar to an article published in a respected journal. Articles from the American Cancer Society website or published in the AORN Journal, by the Association of Operating Room Nurses, should be considered more reliable than a local news organization. Government agencies should also be considered more reliable than many other sources. If a news story is reporting about research published elsewhere, consider using the original source, not the news report. When the reliability of the responsible agent is unknown, other documents from a respected source or website may provide evidence of reliability.

Objectivity and considerations regarding bias may occasionally be a subjective assessment depending on the kind of information needed. The sense of objectivity is often gathered from the tone of language and the belief that the authors of a document are trying to present a balanced discussion of a topic. However, the reader's values, attitudes, feelings, or beliefs affect this perception. An example of different appreciations of objectivity may be observed from reactions to any one of a number of controversial issues. Any given report may be viewed by the reader as biased if it does not provide some level of support for the reader's views. Objectivity is supported in reports of scientific studies when the applicability and usefulness of the research is discussed. Resources produced by companies intending to sell goods or services are often seen as biased but may be useful. Lowered objectivity and higher acceptability of bias may be appropriate if relevancy is high.

Supportive documentation in the form of references, links, and other means for the reader to learn more about the topic helps to authenticate the information being presented. References included in a research report should be of at least the same scholarly level as the report. Internet sources should provide links to other resources on the general topic. Reliability is increased if the references and links are associated with other scholars' works or respected organizations' materials. In some cases there are only a few highly qualified experts in a field, and references to those experts should be expected.

\section{EVALUATION TOOLS}

Websites can be reviewed just like movies and books. Professional journals and organizations will often recommend specific resources. Some information users have found that developing and using a simple checklist tool to maintain a consistent quality of source materials is helpful. An individual can create a personal tool to 
Date assessed: $1 / 5 / 2012$

TOTAL SCORE: $50 / 52$

(Excellent)

Webpage title/URL: AORN, Free Online Education; http://www.aorn.org/FreeCE/

Assessment purpose: Access to free perioperative nursing CEUs for Jones Hospital

Nurses (Hospital Goal 1.2.1)

Scoring: (0) very poor, (1) poor, (2) fair, (3) good, (4) excellent;

Total scores: 0-6 very poor, 7-20, poor, 21-32 fair, 32-45 good, 46-52 excellent

\begin{tabular}{|c|c|c|c|c|c|c|}
\hline \multicolumn{7}{|c|}{ Relevancy } \\
\hline & Very Poor & Poor & Fair & Good & Excellent & Total \\
\hline $\begin{array}{l}\text { Audience clearly identified on } \\
\text { resource }\end{array}$ & & & & 3 & & 3 \\
\hline Audience match to purpose & & & & & 4 & 4 \\
\hline Appropriate language for audience & & & & & 4 & 4 \\
\hline Coverage relative to purpose & & & & & 4 & 4 \\
\hline Timeliness relative to purpose & & & & & 4 & 4 \\
\hline Relevancy: Total Possible 20 & & & & 3 & 16 & 19 \\
\hline
\end{tabular}

\begin{tabular}{|l|l|l|l|l|l|l|}
\hline \multicolumn{5}{|c|}{ Reliability } & \multicolumn{3}{|l|}{} \\
\hline & Very Poor & Poor & Fair & Good & Excellent & Total \\
\hline Author name \& credentials included & & & & 3 & & 3 \\
\hline $\begin{array}{l}\text { Reliable } \\
\text { website/organization/publisher }\end{array}$ & & & & & 4 & 4 \\
\hline Contact information included & & & & & 4 & 4 \\
\hline $\begin{array}{l}\text { Date created/updated } \\
\text { included/published }\end{array}$ & & & & & 4 & 4 \\
\hline Spelling \& grammar correct & & & & & 4 & 4 \\
\hline Information appears accurate & & & & & 4 & 4 \\
\hline Objective report/ bias acceptable & & & & & 4 & 4 \\
\hline Appropriate references, links & & & & 3 & 28 & $\mathbf{3 1}$ \\
\hline \multicolumn{1}{|c|}{ Reliability: Total Possible 32 } & & & & & 4 & 4 \\
\hline
\end{tabular}

\begin{tabular}{|l|l|l|l|l|}
\hline Comments: Assuming nursing CEUs - AORN site \\
Responsible agency assumed from heading \& copyright information \\
Explicit references not necessary at this level; Links work \\
Membership required for some units
\end{tabular}

Fig. 1. Example tool.

systematically evaluate resources. The assessment tool can then be used to compare the quality of a variety of resources (Fig. 1).

The two major categories of a valid assessment are relevancy and reliability. ${ }^{4}$ For webpage assessment, components of relevancy are audience, coverage, timeliness, and language. Components of reliability are accuracy, authority, currency, sponsorship, objectivity, and authentication. An evaluation tool may include these terms as checklist reminders. Assessment can be quantitative or qualitative; a qualitative scale might have related values including not at all, somewhat, reasonable, good, and excellent. An overall assessment would lean toward one value or another. A quantitative assessment of meeting the criteria can be the use of these terms in the form of a 5-point Likert scale, where a value is assigned to each of the points. In the case of quantitative scale, an assigned minimal total score would determine the overall accessibility of the resource. Since each resource is assessed separately relative to meeting some general or specific need, there would need to be one copy of the tool for each resource. An appropriate 
reference to the resource, usually title and retrieval information, date of assessment, and purpose of assessment, should also be included.

The use of an evaluation tool ensures that the same elements are examined consistently. Scanning webpages can be very tiring, and opinions can change based on this fatigue. The personal interests of the evaluator can also alter perceptions of relevancy. Documenting the reason for the evaluation reminds the evaluator of the specific need and required relevancy, as shown in the example in Fig. 1, which shows the focus on perioperative nursing continuing education hours (CE) as opposed to any other specialization. A quantitative assessment, also includes the evaluation points associated with website relevancy and reliability assessments (see Fig. 1). A CE website for a perioperative nurse would have to be current (timeliness) and for nurses (audience). A reliable website that the nurse could use and would use more than once, ideally, should be kept up to date by a recognized, respected organization (author, reliable). To ensure that the website is actually from that organization and not from an unethical entity engaged in a criminal act like information theft, it is important that there be contact information for the organization. Good spelling, grammar, references, and links are significant clues because a respected organization would present itself well and respect the audience. Above all, the assessor has to trust his or her knowledge that the site as a whole makes sense, particularly in terms of information accuracy and bias potential. The consistent reminder to look for these elements ensures that quality resources will be identified because, for example, a good web page can be compared with any other good web page even if the web pages differ in the reasons why they obtained that assessment. Consistency can be even more important when a project extends over long periods of time or when more than one person is involved. If a list of resources is going to be maintained over time, adding dates to reviews is also valuable because organizations change their websites, and universal resource locations (URL) disappear.

\section{SUMMARY}

Several variables should be considered when assessing information sources. Many of these variables are understood and used in interpersonal situations or when using print sources. Assessment of Internet resources has not always been approached in the same manner, but the concepts are transferable. Understanding what information is applicable, who created the source, who or what supports its continuation as a resource, how it can be authenticated, and how it will be used are all critical aspects reliability. The information user's knowledge, combined with systematic consideration of these variables, is needed to ensure the necessary information is gathered and used appropriately. An evaluation tool can be very useful for maintaining assessment consistency over time. ${ }^{1}$

\section{REFERENCES}

1. Rowley J, Hartley R. Organizing knowledge: an introduction to managing access to information. Ashgate Publishing, Ltd; 2006.

2. Ackoff RL. From data to wisdom. J Appl Sys Anal 1989;16:3-9.

3. Wallace DP. Knowledge management: historical and cross-disciplinary themes. Westport (CT): Libraries Unlimited; 2007.

4. Burns N, Grove SK, Gray J. Understanding nursing research: building an evidence based practice. 5th edition. Maryland Heights (MO): Elsevier/Saunders; 2011. 